\title{
Siva's equation for singularity of black holes
}

\author{
Siva Prasad Kodukula \\ Individual Researcher, Q.No.D-11/4,Jindal Nagar, Jindal Steel \& Power Ltd, Angul, Odisha, I N D I A,Pin-759130
}

Email address:

Sivkod@gmail.com

\section{To cite this article:}

Siva Prasad Kodukula. Siva's Equation for Singularity of Black Holes. International Journal of Astrophysics and Space Science. Vol. 1, No. 4, 2013, pp. 16-19. doi: 10.11648/j.ijass.20130104.11

\begin{abstract}
Classical equation for space time and matter gives the relation between a mass and the space time surrounded by its space time fluid. Mass of the basic building block of matter creation has been termed as 'K-Suryon'. Mass of 'K-Suryon' has been calculated as $1.15 \times 10^{-64} \mathrm{kgs}$. The density of its space time fluid is calculated as $3.32 \times 10^{626} \mathrm{Kg} / \mathrm{cum}$. Thus ' $\mathrm{K}$-Suryon' is the most elementary mass that can exist in this universe. We know the minimum mass of anybody to become black hole is 'Chandrasekhar's Limit'. For that mass we can calculate the space time fluid density. So we can find the density of space time fluid associated with it. With these calculations we have concluded that the physical singularity will not be there for a black hole. A physical singularity will exist below a critical density equal to K-Suryon where space time will not exist. It is concluded that velocity of light is reducing and space time fluid density of universe is increasing with the age of Universe. Experimental verification may be possible.
\end{abstract}

Keywords: Singularity, Black Hole, K-Suryon, Space Time Fluid, Universe

\section{Introduction}

Research on 'Heart of the God' model [1] says that universe is existed as it is with some limitations. This is similar result as predicted by 'Steady State Theory' which states that universe is always existed in a steady state, the expansion of the universe is compensated by the continuous creation of matter which is viewed as a property of space, and that despite local evolutionary process, the universe as a whole is not evolving. The rate at which matter is being creating spontaneously is compensating the universe's expansion.

As the surface area of the three- dimensional space time fluid increases, the three-dimensional space will increase. It shows the expansion of universe. But the diameter of the three-dimensional fluid (Heart of the God) is constant. So if its surface area increases its density will decrease so as to compensate expansion. Thus the four dimensional fluid is having a constant radius.

Now let us consider the concept of space time equivalence [3] to understand mass formation from space time and energy. It says that time is nothing but electromagnetic field and also says that the time alone cannot exist in this four dimensional space time continuum. So the time which is more than the least quanta of time i.e $7.6813 \times 10^{-44}$ seconds must have space. It will be in the form of time at this boarder of time and space conversion limit. Above this limit some portion of the time will be converted in to space to form space time. Thus a scope for the existence of gravity will be developed. When this electromagnetic field interacts with another electromagnetic field, the space time associated with it will change its curvature. This is nothing but formation of gravity field. Depending upon the strength of interaction the electromagnetic and gravity fields will exchange their field. As the electromagnetic field reduces, its gravity field will be increased and vice versa. In terms of energy and wave, we can say that an electromagnetic wave loses its frequency without losing its energy. This frequency (equivalent energy) will form gravity field or gravity wave. Now the electromagnetic wave is a combination of electromagnetic field and gravity field. In this interaction when an electromagnetic wave loses its frequency and get converted in to gravity field, the wavelength of electromagnetic wave will increase. But it cannot increase the limit of this universe since its radius is fixed as per 'Heart of God Model'. So exchange will be stopped and the wave will form in to completely mass. This idea is the basis for formation of most elementary particle 'K-Suryon' [1] which is basic building block of all matter. The mass has been calculated as $1.15 \times 10^{-64} \mathrm{kgs}$.

We know that there will be a space time fluid associated with that mass. The density of space time fluid associated for that mass can be calculated by 'Siva's Classical Equation for Space Time and matter' [4]. Now we can find whether that 
mass will float in its own space time fluid or not.

\section{Derivation}

\subsection{Let us Calculate the Space Time Fluid Density Associated with K-Suryon}

We have mass of ' $\mathrm{K}$-suryon ' $=1.15 \times 10^{-64} \mathrm{kgs}$

We have classical equation for space time and matter [4] $\mathrm{M}=\phi \mathrm{d}^{1 / 3}$

Where $\phi=7.06505184 \times 10^{12}$

Where ' $M$ ' is mass and ' $d$ ' is radius of space time density associated with that mass.

So for 'K-Suryon' $\mathrm{d}=4.360329168 \times 10^{-231} \mathrm{mts}$.

We have Siva's Classical Equation for 'space time fluid density' [4] $\gamma \mathrm{d}^{8 / 3}=1.686656885 \times 10^{12}$

Where $\gamma$ is density of space time fluid associated to mass and ' $d$ ' is radius of space time density.

Thus the density of space time fluid associated to K-Suryon can be calculated as $3.3238545 \times 10^{626} \mathrm{~kg} / \mathrm{cum}$

It is the critical density which floats the matter in to space time. Above which the matter will not exist in this space time and there will not exist time bellow this .Here the space starts to convert in to time and matter and pushes the matter back.

\subsection{Let Us Calculate the Space Time Density Associated to the Mass of a Black Hole}

Let us suppose the mass is 'Chandrasekhar's limit' i.e $2.9617698 \times 10^{30} \mathrm{kgs}[1]$

We have classical equation for space time and matter $\mathrm{M}=\mathrm{pd}^{1 / 3}$

Where $\phi=7.06505184 \times 10^{12}$

The radius of space time fluid associated to Black hole can be calculated as $7.3672914 \times 10^{54} \mathrm{mts}$.

We have classical equation for space time fluid density $\gamma \mathrm{d}^{8 / 3}=1.686656885 \times 10^{12}$

If we substitute the above calculated radius value in the equation we can calculate the density of the space time fluid associated to the black hole at Chandrasekhar limit as $1.7682316 \times 10^{-129} \mathrm{~kg} / \mathrm{cum}$.

The Schwarzschild radius of the black hole at Chandrasekhar limit can be calculated as $4.397398294 \times 10^{3}$ mts.

If we consider the mass of the black hole at Chandrasekhar's limit is $2.9617698 \times 10^{30} \mathrm{kgs}$ and radius is Schwarzschild radius as calculated above we can calculate the density of that black hole as $8.315249416 \times 10^{18} \mathrm{Kg} / \mathrm{cum}$.

This density of the black hole with minimum mass is more than its space time fluid density i.e $1.7682316 \times 10^{-129} \mathrm{~kg} / \mathrm{cum}$. So this mass should float in its space time fluid. Then it cannot be a black hole.

In order to remain as a black hole and to satisfy Schwarzschild radius, the radius of space time fluid associated to it should not be more than the Schwarzschild radius. At least it should be equal to it. The excess space time fluid will shrink inside to form a 'singularity' at the center of the black hole. Here the physical existence of singularity is that the mass ' $\mathrm{M}_{\mathrm{S}}$ ' formed due to shrinkage of space time fluid to adjust with the Schwarzschild radius of a black hole of mass ' $\mathrm{M}_{\mathrm{B}}$ ' will be concentrated at the center of the black hole. The point will have zero radius with respect to conventional space time. But it will have a radius in terms of space. Time will not exist inside this singularity.

Let us find the relation between mass of singularity ' $\mathrm{M}_{\mathrm{S}}$ ' and mass of Black hole ' $\mathrm{M}_{\mathrm{B}}$ '

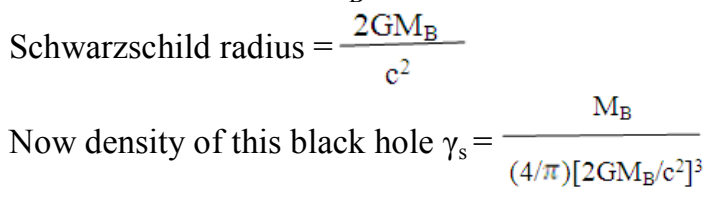

For this $M_{B}$ the mass of singularity is $M_{S}$ The space time fluid density ' $\gamma_{\mathrm{st}}$ ' can be calculated by 'Siva's classical equation for space time and matter' [4]

We have Siva's classical equation for space time and matter [4]

$\mathrm{M}=\mathrm{pd}^{1 / 3}$

Where $\phi=7.06505184 \times 10^{12}$

The equation for a mass ' $\mathrm{M}_{\mathrm{S}}$ ' and 'space time fluid radius ' $\mathrm{d}$ ' is

$\mathrm{M}_{\mathrm{S}}=\phi \mathrm{d}^{1 / 3}$

Where $\phi=7.06505184 \times 10^{12}$

$$
\mathrm{d}^{1 / 3}=\frac{\mathrm{M}_{\mathrm{S}}}{7.06505184 \times 10^{12}}
$$

We have classical equation for space time fluid density [4] $\gamma_{\mathrm{st}} \mathrm{d}^{8 / 3}=1.686656885 \times 10^{12}$

$$
\gamma_{\mathrm{st}}=\frac{1.686656885 \times 10^{12}}{\left(\mathrm{~d}^{1 / 3}\right)^{8}}
$$

If we equate $\gamma_{\mathrm{s}}=\gamma_{\mathrm{st}}$

$$
\begin{aligned}
& \frac{\mathrm{M}_{\mathrm{S}}{ }^{8}}{\mathrm{M}_{\mathrm{B}}{ }^{2}}=\frac{3.12620554 \times 10^{86}}{3 \mathrm{c}^{6}} \\
& \frac{\mathrm{M}_{\mathrm{S}}{ }^{4}}{\mathrm{M}_{\mathrm{B}}}=\frac{1.768107902 \times 10^{43}}{\left[3 \times\left(2.997925 \times 10^{8}\right)^{6}\right]^{12}} \\
& \frac{\mathrm{M}_{\mathrm{S}}{ }^{4}}{\mathrm{M}_{\mathrm{B}}}=3.789207 \times 10^{21}
\end{aligned}
$$

\subsection{The Relation between Singularity of Black Hole and 'K-Suryon'}

K-Suryon is the least mass created in the process of creation of mass. It is the mass created when a wave with a wavelength got stretched and the stretching will be halted due to the limit of this universe as defined by 'Heart of the God model of the universe'. 'Film theory of the universe' [1] says that the universe is made up of films and the films will be changed for every $7.6813002 \times 10^{-44} \mathrm{sec}$. It says that there will not exist time below this limit. 'Space time equivalence concept' describes the formation of space time .The mass will be formed between this film change. So mass bellow 'K-Suryon' will not exist. Space time also will not exist. So we can say this limit is the point of singularity of space time. 
But below this, gravity also will not exist since there is no space time. Thus, this mass can be treated as a black hole with 'plank mass'

But as calculated above the space time fluid density of mass of K-Suryon is more than the density of K-Suryon treated as a black hole (volume calculated by Schwarzschild radius of mass of K-Suryon). It is not possible because its space time will be inside the mass .For a black hole, the space time density of the mass should be less than its density.

Now we have to suppose the black hole created by mass of K-Suryon will contain the mass concentrated at a center called singularly. Now we can calculate mass of this singularity as described by the equation (1) .This must be equal to plank mass of quantum mechanics. Since it obeys both the conditions -limit to the length of wave length formed while creation of mass and the mass created to form black hole.

If we substitute $M_{B}$ with mass of K-suryon, we found the mass of singularly as $8.1322214 \times 10^{-10} \mathrm{kgs}$

This is not equal to plank mass.

So if we go back to the calculation of 'Mass of K-suryon'

The wavelength limit is equivalent length of dia of heart of God.i.e calculated by the equation $\mathrm{d}=\mathrm{H} / \mathrm{V}_{\text {minimum }}$; where $\mathrm{H}=$ Hubble's constant and $\mathrm{V}_{\text {minimum }}=1000 \mathrm{mts} / \mathrm{sec}$ as per 'Double Relativity'.

As per mass energy equation and plank equations$(\mathrm{hc} / \lambda)=\mathrm{m}_{\mathrm{k}} \mathrm{c}^{2}$ or $\mathrm{m}_{\mathrm{k}}=(\mathrm{h} / \lambda \mathrm{c})$ Where $\mathrm{h}$ is planks constant , $\mathrm{c}$ is velocity of light, $\mathrm{m}_{\mathrm{k}}$ is mass of $\mathrm{K}$-suryon and $\lambda=$ dia of Heart of God i.e. 'd'

Here we can doubt the calculation of Heart of God. That means on $V_{\text {minimum }}$ and ' $H$ '. So let us not put the value of ' $d$ ' in the place of $\lambda$.

Let us substitute $\mathrm{V}_{\text {minimum }} / \mathrm{H}$ in place of $\lambda$

Now $\lambda=\mathrm{M}_{\mathrm{k}}=\frac{\mathrm{hH}}{\mathrm{c} \mathrm{V} \text { min }}$

We have equation (1)

If we substitute $m_{k}$ in the place of $M_{B}$

We can rewrite the equation as

$$
\begin{aligned}
& \frac{\mathrm{M}_{\mathrm{S}}{ }^{4}}{\mathrm{~m}_{\mathrm{k}}}=3.789207 \times 10^{21} \\
& \frac{\mathrm{M}_{\mathrm{s}}{ }^{4} \mathrm{~V}_{\min } \times \mathrm{c}}{\mathrm{h} \times \mathrm{H}}=3.789207 \times 10^{21}
\end{aligned}
$$

As explained above the mass $\mathrm{M}_{\mathrm{S}}$ must be equal to the plank mass.

Therefore $\mathrm{M}_{\mathrm{S}}=\sqrt{ }(\mathrm{hc} / \mathrm{G})$

If we substitute this value in the above equation

$$
\begin{aligned}
& \frac{\mathrm{h}^{2} \mathrm{c}^{2} \times V_{\min } \times c}{\mathrm{G}^{2} \mathrm{hxH}}=3.789207 \times 10^{21} \\
& \frac{\mathrm{hxc^{3 }}}{\mathrm{G}^{2}} \times \frac{\mathrm{V}_{\min }}{\mathrm{H}}=3.789207 \times 10^{21}
\end{aligned}
$$

We know that $\frac{\mathrm{V}_{\min }}{\mathrm{H}}=\mathrm{d}$

Where ' $d$ ' is the diameter of the universe at the
Beginning of expansion.

Thus we can find an equation showing the relation between c, G,h and dia of the universe ' $\mathrm{d}$ '

$$
\mathrm{dc}^{3}=3.789207 \times 10^{21}\left(\mathrm{G}^{2} / \mathrm{h}\right)
$$

If we substitute the values of $c, G$ and $h$ we can get the value of $\mathrm{d}=9.4 \times 10^{8} \mathrm{mts}$.

But we have $\mathrm{d}=\frac{\mathrm{V}_{\min }}{\mathrm{H}}=1.9147644 \times 10^{22} \mathrm{mts}$.

Implies, the dia of this universe at the beginning is $1.9147644 \times 10^{22} \mathrm{mts}$ and at present is $9.4 \times 10^{8} \mathrm{mts}$. Implies, the distance is reducing as the age of the universe increases.

If we consider that light velocity ' $c$ ' is constant in this universe then

$\mathrm{c}=$ distance $/$ time

The distance is changing with the age of universe. Then the time is also changing with the age of universe.

The unit length and unit time are changing and the velocity of light is constant and should be $2.997925 \times 10^{8}$ $\mathrm{mts} / \mathrm{sec}$. But here the distance which has to become $2.997925 \times 10^{8} \mathrm{mts}$ has become only $9.4 \times 10^{8} \mathrm{mts}$. So the light has taken long time to cover this distance in one second. In other terms, it seems that light velocity has been reduced. Why this reduction occurs in light velocity? It may be due to that reason that the medium which is space time fluid for light is becoming denser with the age of the universe.

\section{Conclusions}

1. Singularity of a black hole can be expressed in terms of mass $\left(\mathrm{M}_{\mathrm{S}}\right)$ even though it does not contain mass or space. Here space time conversion plays major role.

2. There is a relation between Mass of Black hole $\left(M_{B}\right)$ and Mass of its Singularity $\left(\mathrm{M}_{\mathrm{S}}\right)$.

The equation is -

$$
\frac{\mathrm{M}_{\mathrm{S}}{ }^{4}}{\mathrm{M}_{\mathrm{B}}}=3.789207 \times 10^{21}
$$

3. The equation $\mathrm{dc}^{3}=3.789207 \times 10^{21}\left(\mathrm{G}^{2} / \mathrm{h}\right)$

Shows the relation between dia of the Universe ' $d$ ', velocity of light 'c' Gravitational constant ' $G$ ' and planks constant ' $h$ '.

4. This concept concluded that velocity of light is decreasing with expansion of the universe. Present velocity of time is about 3.13 times reduced from commencement of its expansion.

\section{References}

[1] S.P.Kodukula,Heart of the God with Grand proof Equation A classical approach to quantum theory (ISBN 978-0-557-08995-6).Raleigh,NorthCorolina:Lulu.com, pp3-8,31-53,79-93,2009.

[2] S.P.Kodukula, “ New Cosmological Constants", ID no.1,FFP9,2008http://agenda.fisica.uniud.it/difa/contributio nDlay.py? contribId $=1$ \&amp; confld $=9$ accessed on $17^{\text {th }}$ Aug 2013. 
[3] S.P.Kodukula, "Space Time Equivalence-A New Concept". International journal of Scientific Research and Publications,vol.2,No.10,pp.1-3,Oct.2012.

[4] S.P.Kodukula, "Siva's Classical Equation for Space Time and Matter"., International Journal of Advancements in Research \& Technology', Volume 2, Issue8, August-2013 Web link: http://www.IJoART.org

[5] S.P.Kodukula, "Derivation of Siva's Constant ' $\mathrm{K}$ ' of
Physics"., International Journal of Advancements in Research \& Technology', Volume 2, Issue1, January-2013 Web link: http://www.IJoART.org

[6] S.W.Hawking , A Brief History of time'. Bantam press,pp32-36,1988.

[7] S.W.Hawking \& R.Penrose, The Nature of space and Time, Princeton University press, pp.116- 118,1998 